\title{
Softening up on the hardening hypothesis
}

Joanna E Cohen, ${ }^{1,2}$ Paul W McDonald, ${ }^{2,3}$ Peter Selby ${ }^{4}$

The hardening hypothesis has intuitive and common sense appeal: in jurisdictions that have implemented evidence-based tobacco control policies, the smokers who have a relatively easy time quitting will quit, and as the future unfolds there will be an increasing proportion of remaining smokers who cannot quit and are more resistant to quitting than smokers in the past.

In this context, 'hardening' is a measure of a group, or population, over time. If a group or population is hardening, it implies that the proportion of smokers who are 'hard core' is increasing.

'Hard core' was first used in the peer reviewed literature in relation to smokers by Lichtenstein and Keutzer in 1973, in their review of how psychological research could be applied in smoking cessation clinics. ${ }^{1}$ Following this initial intimation of a hard core smoker, the term popped up again in the late $1980 \mathrm{~s},{ }^{2-5}$ and since then the literature on hard core smokers has grown, although the number of papers that have empirically examined this topic remains limited.

The bottom line from the body of evidence to date is that smokers classified as hard core represent only a very small minority of all smokers (in selected high income countries for which data have been available), and that 'hardening of the target' is still a long way off. Even analyses focused on the individual level find only a handful of subgroups where there is a suggestion of hardening. Cross-sectional data show that the lower the prevalence of smoking, the lower the average number

${ }^{1}$ Institute for Global Tobacco Control, Department of Health, Behavior and Society, Johns Hopkins Bloomberg School of Public Health, Baltimore, USA; ${ }^{2}$ Ontario Tobacco Research Unit, Toronto, Waterloo, Canada; ${ }^{3}$ School of Public Health and Health Systems, University of Waterloo, Waterloo, Canada; ${ }^{4}$ Centre for Addiction and Mental Health, Addictions Program, Toronto, Ontario, Canada

Correspondence to Dr Joanna E Cohen, Director, Institute for Global Tobacco Control, Bloomberg Associate Professor of Disease Prevention, Johns Hopkins Bloomberg School of Public Health, $624 \mathrm{~N}$ Broadway, Hampton House 297, Baltimore, MD 21205-1996, USA; jocohen@jhsph.edu of cigarettes smoked per day and the lower the percentage of smokers who smoke within $30 \mathrm{~min}$ of waking. ${ }^{6}$ Similarly, perceived self-efficacy for quitting, intentions to quit and the proportion of smokers who are able to remain abstinent for 3 months are all higher at lower levels of smoking prevalence. ${ }^{6}$ Warner and Burns reviewed US data from the 1990s and found that the population of smokers continued to be dominated by quittingsusceptible individuals and that cessation rates had not decreased over the decade; they concluded that, at the time of their analysis, hardening had not occurred at the population level. ${ }^{7}$

In a more recent comprehensive review of the empirical literature on hardening published in 2011, Hughes reviewed the literature exploring the relationship between nicotine dependence and the ability to remain abstinent. ${ }^{8}$ His updated synthesis continued to find that there was no increase in cigarettes per day and time to first cigarette; while Diagnostic and Statistical Manual of Mental Disorders (DSM)-defined dependence did appear to be increasing over time, these two studies did not explore whether this measure of dependence was related to the ability to quit. Hughes concluded that although there is a suggestion that hardening is occurring among treatment seekers, there remains no clear evidence of hardening in the general population.

One cannot help join Hughes in being somewhat surprised that the common sense and intuitive hardening hypothesis is not (yet?) supported by the empirical literature. Hughes' solution is to conduct more and better studies, with improved measures of dependence, quit attempts and quit attempt success. ${ }^{8}$ In contrast, when we take stock of what is known and what the implications are of this knowledge, we instead propose that it is time to make further investments in effective strategies to help smokers stop and stop for good.

In the final analysis, knowing whether the population of smokers is hardening or not will not have bona fide implications for what needs to be done to reduce tobacco-caused death and disease, at least in the short-term and mid-term. It is imperative to note that both populationbased and individual-based interventions have not yet been used to their full advantages. Our efforts need to focus on how to increase the collective effectiveness, reach, adoption, cost efficiency and benefit of these complementary sets of interventions.

For example, with respect to population-based interventions, tobacco products in many jurisdictions remain inexpensive. In addition, tobacco products continue to be hyper-available, being sold widely in gas stations, convenience stores and grocery stores with no restrictions on either the absolute number or the density of these retail outlets. Tobacco remains affordable. Few jurisdictions have set minimum prices or maximised other price-based measures. Overt advertising and promotion of tobacco products remain, at a minimum through direct mailings to smokers, through publications with a predominantly adult readership and through smoking in the media including movies. Progress towards reducing people's exposure to secondhand smoke in public places has been remarkably swift; however, even in jurisdictions with comprehensive smoke-free laws or regulations, people continue to be exposed, for example, in multi-unit dwellings. Tobacco products themselves are still attractive through their increasingly sophisticated packaging and through formulations that make products more palatable. A handful of jurisdictions have implemented well designed mass media campaigns, but only a small minority has taken a comprehensive approach by linking television and radio advertisements with package warnings, quit lines that offer both counseling and medication and printed and electronic educational materials. All of these evidence-based interventions need to be continuously maintained at a sufficient dose.

Treatment interventions also have not been used to their full potential. Despite the existence of evidence-based clinical practice guidelines, and significant promotion of cessation treatments in some jurisdictions, widespread appropriate adoption of behavioural and pharmacological treatment has been limited. ${ }^{9} 10$ Adoption by smokers has been low even in jurisdictions where barriers such as cost have been removed. ${ }^{9}$ The view by many practitioners that tobacco use is a relatively low therapeutic priority is another barrier; should the patient suffer from comorbidities, smoking 
cessation often takes a back seat to other presenting problems, although it often negatively affects the outcome. ${ }^{11}$ Moreover, nicotine replacement therapy (NRT) is recommended for use in fixed doses, with appropriate guidelines for combination therapy lacking. As we focus on increasing the implementation and reach of our existing interventions, the search for novel methods to achieve smoking cessation must continue. ${ }^{12}$

Insistence that individual smokers are becoming more resistant to quitting and that populations are hardening is reminiscent of victim blaming. Victims of conditions such as tobacco use and addiction suffer from terrible tobaccorelated illnesses and must endure the spectre of being blamed, marginalised and derogated. ${ }^{13}$ Victim blaming manipulates attributions of responsibility. ${ }^{14}$ Crawford suggests victim blaming is used to justify restrictions in access to medical services and to divert attention from social determinants as well as the failure of commercial products and services. ${ }^{15}$

In his recent review, Hughes presents a heuristic model of smoking cessation, ${ }^{8}$ which he uses as the basis for his analysis and discussion of hardening. However, his model treats tobacco control policies solely as a driver of quit attempts, with no impact on the ability to maintain abstinence following a quit attempt. Population-level tobacco control interventions (such as those that increase price, reduce availability, eliminate advertising and promotion, protect people from secondhand smoke, reduce the attractiveness of tobacco products, and effectively inform the public about the impacts of these products) will continue to be important: not only is there a need to address the continuous cycle of new cohorts at risk of entering the market, ${ }^{16}$ but these interventions can also support the ability to quit and stay quit.

Humans do not need to consume tobacco products for survival and therefore, theoretically, it is possible to eventually get tobacco use down to very, very low rates of use. Regardless of the situation a country is in with regard to this epidemic and implementation of tobacco control interventions, the focus should be on a comprehensive approach to tackling this wholly preventable yet devastating cause of death and disease-an approach that implements and sustains the gamut of both population-based and treatmentoriented interventions. As researchers and practitioners, we must resist the lure of the as yet unproven hardening hypothesis and instead concentrate our efforts on improving the effectiveness of the full range of tobacco control interventions.

Competing interests PS has received funds from manufacturers of smoking cessation medications (Pfizer Inc, Johnson and Johnson) for research, speaker fees and consultation. He is a member of an independent Data Safety and Monitoring Board for NABI pharmaceuticals, the manufacturer of a nicotine vaccine.

Provenance and peer review Commissioned; externally peer reviewed.

Tobacco Control 2012;21:265-266.

doi:10.1136/tobaccocontrol-2011-050381

\section{REFERENCES}

1. Lichtenstein $\mathbf{E}$, Keutzer CS. Implications of psychological research for smoking control clinics. Health Serv Rep 1973;88:535-40.
2. Nevin N, Lynch $M$, Kropf G, et al. Helping the hard core smoker. Can Nurse 1987;83:20-2.

3. DiFranza JR, Guerrera MP. Hard-core smokers JAMA 1989;261:2634-5.

4. Ginne M. Tertiary-level interventions for hardcore smokers. J Psychoactive Drugs 1989;21:343-53.

5. Pierce J, Giovino G, Hatziandreu E, et al. National age and sex differences in quitting smoking. $J$ Psychoactive Drugs 1989;21:293-8.

6. Giovino G. Unassisted Quitting Vs. Cessation Interventions in the Era of Tobacco Regulation and Health Care Reform. Symposium at the $17^{\text {th }}$ Annual Meeting of the Society for Research on Nicotine and Tobacco. Toronto: Canada, 2011.

7. Warner KE, Burns DM. Hardening and the hard-core smoker: concepts, evidence, and implications. Nicotine Tob Res 2003;5:37-48.

8. Hughes JR. The hardening hypothesis: is the ability to quit decreasing due to increasing nicotine dependence? A review and commentary. Drug Alcohol Depend 2011;117:111-17.

9. Burns ME, Rosenberg MA, Fiore MC. Use of a new comprehensive insurance benefit for smoking-cessation treatment. Prev Chronic Dis 2005;2:A15.

10. Fiore MC, Keller PA, Curry SJ. Health system changes to facilitate the delivery of tobaccodependence treatment. Am J Prev Med 2007;33 (Suppl 6):S349-56.

11. Tajima B, Guydish J, Delucchi K, et al. Staff knowledge, attitudes, and practices regarding nicotine dependence differ by setting. J Drug Issues 2009;39:365-84.

12. Bader P, McDonald P, Selby P. An algorithm for tailoring pharmacotherapy for smoking cessation: results from a Delphi panel of international experts. Tob Control 2009;18:34-42.

13. Ryan W. Blaming the Victim. New York: Pantheon, 1971.

14. Maes J. Blaming the victim: belief in control or belief in justice? Soc Justice Res 1994;7:69-90.

15. Crawford R. You are dangerous to your health: the ideology and politics of victim blaming. Int $\mathrm{J}$ Health Serv 1977;7:663-80.

16. Chaiton MO, Cohen JE, Frank J. Population health and the hardcore smoker: Geoffrey Rose revisited. $J$ Public Health Policy 2008;29:307-18. 